\title{
A New Immunofluorescence Assay for Fecal Calprotectin Distinguishes Inflammatory Bowel Disease from Functional Bowel Disease
}

\author{
Lixia Xu1", Peisi Rao1\#, Xin Liu² ${ }^{2}$ Zhirong Zeng1 ${ }^{*}$ \\ ${ }^{1}$ Department of Gastroenterology and Hepatology, The First Affiliated Hospital of Sun Yat-sen University, \\ Guangzhou, China \\ ${ }^{2}$ Zhongshan School of Medicine, Sun Yat-sen University, Guangzhou, China \\ Email: *zengzhirong@vip.163.com
}

How to cite this paper: Xu, L.X., Rao, P.S. Liu, X. and Zeng, Z.R. (2018) A New Immunofluorescence Assay for Fecal Calprotectin Distinguishes Inflammatory Bowel Disease from Functional Bowel Disease. Open Journal of Gastroenterology, 8, 405-414.

https://doi.org/10.4236/ojgas.2018.811042

Received: October 28, 2018

Accepted: November 12, 2018

Published: November 15, 2018

Copyright $\odot 2018$ by authors and Scientific Research Publishing Inc. This work is licensed under the Creative Commons Attribution International License (CC BY 4.0).

http://creativecommons.org/licenses/by/4.0/ Open Access

\begin{abstract}
Aims: To investigate the diagnostic value of fecal calprotectin (FC) determined by a new immunofluorescence assay-fluorescence enzyme immunoassay (FEIA) in patient with inflammatory bowel disease (IBD) or functional bowel disease, compared with the typical ELISA kit. Methods: FC was determined simultaneously by FEIA and an ELISA kit in 26 patients with functional bowel disease and 77 patients with IBD. We compared the difference of FC levels between patients with IBD and patients with functional bowel disease. Receiver operating characteristics curve (ROC) was constructed to obtain the optimal cut-off value of FC for distinguishing IBD from functional bowel disease and the corresponding sensitivity and specificity. Results: The median FC levels of patients with IBD in clinical active stage or clinical remission stage was significantly higher than that of patients with functional bowel disease. The median FC levels of patients with IBD in clinical active stage, IBD in clinical remission stage and functional bowel disease were as follow: 699.91 (346.14 1647.54) $\mu \mathrm{g} / \mathrm{g} ; 407.36$ (121.81 878.48) $\mu \mathrm{g} / \mathrm{g} ; 39.04$ $(12.09 \sim 81.04) \mu \mathrm{g} / \mathrm{g}$ when FC was measured by FEIA. The median FC levels were $716.99(240.42 \sim 1232.53) \mu \mathrm{g} / \mathrm{g} ; 338.46(53.08 \sim 692.82) \mu \mathrm{g} / \mathrm{g} ; 41.44$ $(11.77 \sim 73.19) \mu \mathrm{g} / \mathrm{g}$ among such above three groups of patients respectively, when FC was measured by ELISA kit. The diagnostic value of IBD with FC determined by FEIA (optimal cut-off $=131.79 \mu \mathrm{g} / \mathrm{g}$ ) and ELISA kit (optimal cut-off $=121.85 \mu \mathrm{g} / \mathrm{g}$ ) presented an area under the curve of 0.881 and 0.873 , respectively. Conclusions: FC determined by FEIA was an accurate surrogate marker to distinguish IBD from functional bowel disease.
\end{abstract}

\section{Keywords}

Inflammatory Bowel Disease, Functional Bowel Disease, Fecal Calprotectin 


\section{Introduction}

Inflammatory bowel disease (IBD) is a group of non-specific chronic intestinal inflammation of unknown etiology, including ulcerative colitis (UC) and Crohn's disease (CD). In recent years, the incidence of IBD in China has increased significantly [1] [2] [3]. The major symptoms of IBD are abdominal pain, diarrhea, bloody mucopurulent stool and weight loss. At the early stage, most IBD patients only have manifestations similar to functional bowel disease, such as mild diarrhea and abdominal pain, making it difficult for differential diagnosis. The current differential diagnosis of IBD and functional bowel disease depends on endoscopy. However, endoscopy is an invasive examination that patients may have complications such as perforation, hemorrhage, and infection. In addition, patients have to take laxatives to clean the intestines before examination, which is painful with low acceptance. Therefore, it is in urgent need to find a non-invasive indicator of intestinal inflammation to help clinicians quickly distinguish IBD from functional bowel disease.

Calprotectin is a calcium-binding protein of the S100 protein family. It is mainly from neutrophils, and a small part was found in monocytes and reactive macrophages [4], accounting for approximately $60 \%$ of the total cytosolic protein in neutrophils, so it can be used as a marker of inflammatory activation. Many studies have found that fecal calprotectin (FC) plays an important role in the identification of IBD and functional bowel disease [5] [6].

We have developed a new automatic FC detection kit using fluorescence enzyme immunoassay (FEIA) which can quickly and accurately detect FC in a short time. In this study, we aimed to evaluate the assay in the differential diagnosis between IBD and functional bowel disease, and further to explore whether it can be used as an important screening test.

\section{Materials and Methods}

\subsection{Participants}

During the period between from April 2014 to December 2014, consecutive 77 patients with IBD ( $\mathrm{n}=40$ for $\mathrm{CD}, \mathrm{n}=37$ for $\mathrm{UC}$ ) undergoing colonoscopy, who were diagnosed at IBD Center, The First Affiliated Hospital of Sun Yat-sen University, were included in this study. IBD diagnosis for all patients was established with endoscopic and histological criteria at least 6 months before inclusion, according to the IBD diagnostic criteria recommended by the Society of Gastroenterology of the Chinese Medical Association in 2012 [7]. Patients with the drug history of non-steroidal anti-inflammatory drug (NSAID) or proton pump inhibitor (PPI) within previous three months, surgery within previous three months, alcohol abuse, combined gastrointestinal infections, gastrointestinal cancer, pregnancy, combined severe systemic disease of heart, lung, kidney and brain, or indeterminate colitis were not included. Meanwhile this study also included 26 patients, who visited our outpatient department or were admitted to our hospital during the same period, with gastrointestinal symptoms such as abdominal pain, 
diarrhea and constipation, but the result of colonoscopy were normal and other gastrointestinal diseases were excluded as the control group (i.e., patients with functional bowel disease). The stools of the two groups were collected.

The study was approved by the Clinical Research Ethics Committee of the First Affiliated Hospital of Sun Yat-sen University, and the specimens were collected with informed consents from the participants.

\subsection{Sample and Data Collection}

10 - $20 \mathrm{~g}$ stool sample was collected within 1 week before and after the endoscopy examination. All samples were sent and stored at $-80^{\circ} \mathrm{C}$ within 24 hours. Stool sample would not be collected within 1 day after the patient took laxatives for colonoscopy examination. The demographic and clinical data such as clinical symptoms and laboratory tests of participants were also collected.

\subsection{Fecal Calprotectin Determination}

In this study, the immunofluorescence kit developed by our hospital and the PhiCal ${ }^{\oplus}$ Calprotectin ELISA Kit (K6927, Germany) were used to simultaneously detect the FC in the samples. The FC content was measured in strict accordance with the kit instructions. If the result could not be measured by diluting the sample at a ratio of 1:50, the dilution ratio could be further increased until it is measured. If a negative value occurred, it would be considered that $\mathrm{A}$ value of the sample was lower than that of the blank, indicating that FC content of the sample was very low, and the concentration of these samples would be uniformly set to zero.

The PhiCal Calprotectin ELISA Kit costs 5000 RMB and takes about 6 hours to make 98 samples at a time. Compared with ELISA, the FC immunofluorescence detection method can detect one stool sample alone and only needs about 15 - 20 minutes; besides a special immunofluorescence measuring instrument, it does not need a special laboratory and professional laboratory technicians. It is simple to operate and all operations are performed by the instrument.

\subsection{Statistics Analysis}

Statistical analysis was performed using Medcalc 14.8.1 statistical software. Measurement data that agreed with the normal distribution were expressed with mean \pm standard deviation, and $t$ test comparison was used between the two groups. Measurement data that did not agree with the normal distribution were expressed with median and interquartile range (IQR). Wilcoxon test was used to compare continuous variables which were divided into two groups, and Kruskal-Wallis was used to compare continuous variables which were divided into more than two groups. If Kruskal-Wallis result showed the groups differed and the average rank sum was further compared using the Bonferroni method. Receiver operating characteristics (ROC) analyses were carried out to determine the test characteristics of FC in the differential diagnosis of IBD and functional 
bowel disease. Optimal cut-off values have been calculated based on the highest sum of sensitivity and specificity. In addition, the corresponding sensitivity, specificity and negative predictive value (NPV), positive-predictive value (PPV), negative-likelihood ratios (LR-), positive-likelihood ratios (LR+), and area under the curve (AUC) were calculated. $P<0.05$ was considered statistically significant.

\section{Results}

\subsection{Patient Data}

A total of 103 patients were enrolled in the study, including 26 in the control group and 77 in the IBD group. The baseline characteristics of enrolled patients are shown in the Table 1 . The average age of the IBD group was lower than that of the control group, and the average age of the $\mathrm{CD}$ group was lower than that of the UC group and the control group $(p=0.001)$.

\subsection{Comparison of FC in Patients with IBD or Functional Bowel Disease}

In the normality test, FC concentration does not conform to the normal distribution, so it is expressed by the median and interquartile range. Through using FEIA, the median FC concentration of the control group was 39.04 (12.09 81.04) $\mu \mathrm{g} / \mathrm{g}$, and the median FC concentration of IBD in remission stage was 407.36 (121.81 878.48) $\mu \mathrm{g} / \mathrm{g}$, while the median FC concentration of active IBD was $699.91(346.14 \sim 1647.54) \mu \mathrm{g} / \mathrm{g}$. There were significant differences between IBD in active stage $v s$ control group, IBD in remission stage $v s$ control group ( $p$ $<0.0167)$. Detected by ELISA, the median FC concentration of the control group was $41.44(11.77 \sim 73.19) \mu \mathrm{g} / \mathrm{g}$, and the median FC concentration of the IBD in remission stage was 338.46 (53.08 - 692.82) $\mu \mathrm{g} / \mathrm{g}$, while the median FC concentration of active IBD was $716.99(240.42 \sim 1232.53) \mu \mathrm{g} / \mathrm{g}$. There were significant differences between active IBD group $v s$ remission IBD group, active IBD group $v s$ control group, remission IBD group vs control group $(p<0.0167)$. The results were shown in Figure 1. Further subgroup analysis revealed there was no statistical difference between FC concentration in the IBD group during clinical remission stage, mild activity, moderate activity, and severe activity measured by these two detection methods.

\subsection{Mapping the ROC Curve to Analyze the Differential Diagnosis Ability of FC for IBD and Functional Intestinal Diseases}

In the ROC curve analysis, there was no statistical difference between the AUC values of the FEIA and the ELISA method, with the AUC values of 0.881 and 0.873 respectively. If the cut-off values of FC concentration change, its specificity and sensitivity for differential diagnosis of IBD and functional bowel disease were different. By using FEIA method, the Youden coefficient was the largest when the optimal cut-off value of FC concentration was $131.79 \mu \mathrm{g} / \mathrm{g}$, and the 
Table 1. Baseline characteristics of enrolled patients.

\begin{tabular}{|c|c|}
\hline $\mathrm{N}$ (cases) & 103 \\
\hline IBS & 26 \\
\hline $\mathrm{CD}$ & 40 \\
\hline UC & 37 \\
\hline \multicolumn{2}{|l|}{ Age(years) } \\
\hline IBS & $45.00 \pm 16.00$ \\
\hline IBD & $34.74 \pm 15.61$ \\
\hline $\mathrm{CD}$ & $25.30 \pm 10.08$ \\
\hline UC & $44.94 \pm 14.09$ \\
\hline \multicolumn{2}{|l|}{ Male gender(cases) } \\
\hline IBS & $12(46.15 \%)$ \\
\hline IBD & $48(62.34 \%)$ \\
\hline $\mathrm{CD}$ & $26(65.00 \%)$ \\
\hline UC & $22(59.46 \%)$ \\
\hline \multicolumn{2}{|l|}{ FC by ELISA $(\mu \mathrm{g} / \mathrm{g})$} \\
\hline IBS & $41.44(11.77 \sim 73.19)$ \\
\hline IBD in clinical remission stage & $338.46(53.08-692.82)$ \\
\hline IBD in clinical active stage & $716.99(240.42 \sim 1232.53)$ \\
\hline \multicolumn{2}{|l|}{$\operatorname{FC}$ by $\operatorname{FEIA}(\mu \mathrm{g} / \mathrm{g})$} \\
\hline IBS & $39.04(12.09 \sim 81.04)$ \\
\hline IBD in clinical remission stage & $407.36(121.81 \sim 878.48)$ \\
\hline IBD in clinical active stage & $699.91(346.14 \sim 1647.54)^{*}$ \\
\hline
\end{tabular}

${ }^{*}$ There were significant differences between IBD in active stage vs control group, IBD in remission stage vs control group $(\mathrm{p}<0.0167)$.

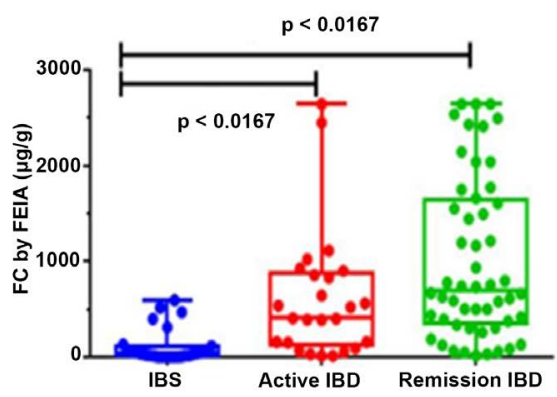

(a)

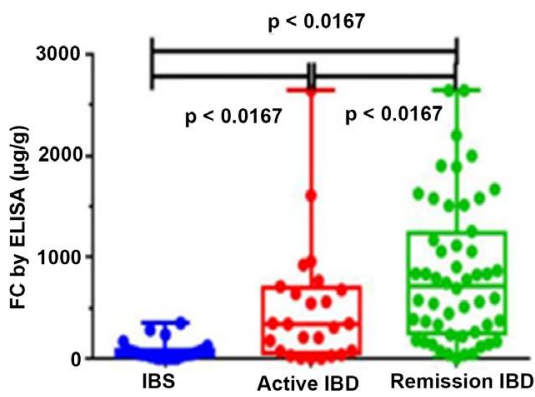

(b)

Figure 1. Box plot with scatter diagram of FC detected by FEIA and ELISA in the control group, the active IBD group and the remission IBD group. (a) The results of the FEIA method; (b) The results of the ELISA method.

corresponding sensitivity and specificity were $81.82 \%$ and $80.77 \%$ respectively. Through using the ELISA, the optimal cut-off value of FC concentration was $121.85 \mu \mathrm{g} / \mathrm{g}$, the sensitivity was $81.82 \%$, and the specificity was $84.62 \%$. The results were shown in Table 2 and Figure 2. 
Table 2. ROC curve analysis of FC measured by ELISA and FEIA.

\begin{tabular}{ccc}
\hline & ELISA & FEIA \\
\hline AUC & 0.873 & 0.881 \\
Standard deviation & 0.034 & 0.035 \\
95\% confidence interval & $0.793-0.931$ & $0.803-0.937$ \\
Cut-off value $(\mu \mathrm{g} / \mathrm{g})$ & 124.85 & 131.79 \\
Sensitivity & $81.82 \%$ & $81.82 \%$ \\
Specificity & $84.62 \%$ & $80.77 \%$ \\
Positive predicted value (PPV) & $94.0 \%$ & $92.6 \%$ \\
Negative predicted value (NPV) & $61.1 \%$ & $60.0 \%$ \\
Positive likelihood ratio (LR+) & 5.32 & 4.25 \\
Negative likelihood ratio (LR-) & 0.21 & 0.23 \\
\hline
\end{tabular}

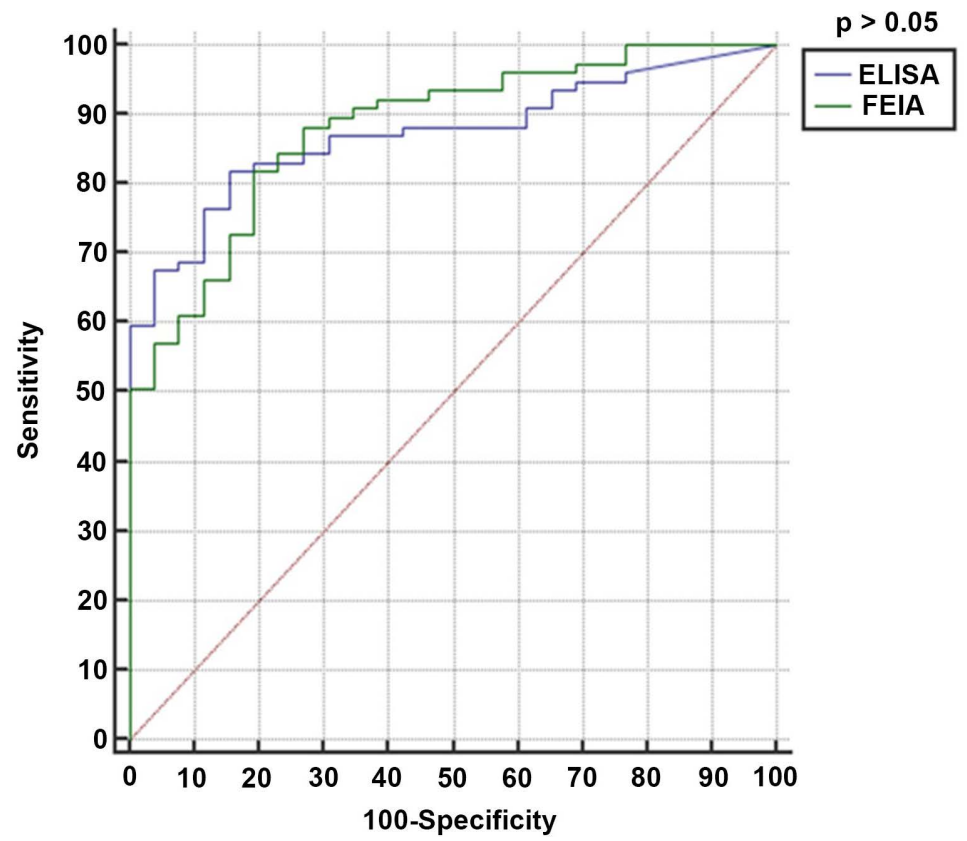

Figure 2. ROC curve analysis of FC measured by ELISA and FEIA. There was no statistical difference between the AUC values of the FEIA and the ELISA method, with the AUC values of 0.881 and 0.873 respectively. By using FEIA method the corresponding sensitivity and specificity were $81.82 \%$ and $80.77 \%$ respectively. Through using the ELISA, the sensitivity was $81.82 \%$, and the specificity was $84.62 \%$.

\subsection{Comparison of the Positive Rates of FEIA and ELISA}

According to the results of ROC curve analysis, the cut-off value of the FEIA method was set to $131.79 \mu \mathrm{g} / \mathrm{g}$, and the cut-off value of the ELISA method was set to $121.85 \mu \mathrm{g} / \mathrm{g}$. When FEIA was used to measure FC concentration, the positive rate of functional bowel disease was $15.38 \%(4 / 26)$, and the positive rate of IBD group was $81.82 \%$ (63/77). Though using ELISA to detect FC concentration, 
the positive rate of functional bowel disease was $11.54 \%$ (3/26), and the positive rate of IBD group was $81.92 \%$ (63/77). Among the 14 patients who failed to be identified as IBD by FEIA, 9 of them could not be identified by ELISA, neither. FC levels of 4 patients with functional bowel disease were greater than 131.79 $\mu \mathrm{g} / \mathrm{g}$ in FEIA, and 3 of them were also greater than the cut-off value of ELISA $(121.85 \mu \mathrm{g} / \mathrm{g})$ in ELISA test. There was no significant difference in the overall positive rate of FC concentration measured by the two test methods $(p>0.05)$. The Kappa value was 0.724 .

\section{Discussion}

IBD mainly includes CD and UC. Clinical manifestations of IBD overlap with functional bowel disease such as irritable bowel syndrome (IBS). It is reported that about $10 \%-20 \%$ of the general population complaining of abdominal discomfort and other gastrointestinal symptoms were eventually diagnosed as IBS, about $40 \%$ of IBD patients met the IBS Rome III diagnostic criteria [8], the life quality of $1 / 3$ remission UC patients and about $42 \%$ CD patients was seriously decreased due to the presence of IBS symptoms [9]. This brings great difficulties to the clinician's differential diagnosis, so it is often necessary to arrange for colonoscopy to identify patients with similar symptoms of IBS or IBD disease. More importantly, treatment of IBD is completely different from that of functional bowel disease such as IBS, so it is of great significance for clinicians to quickly, easily and effectively identify these diagnoses from the digestive tract symptoms.

In 1999, Roseth AG found that FC was proportional to the indium-111-labelled leukocytes in the feces, suggesting that FC was highly correlated with neutrophils migration and permeation in the digestive tract, and can specifically respond to gastrointestinal inflammation [4]. Subsequently, it was gradually discovered that FC can be used to distinguish organic intestinal disease (such as IBD) from functional gastrointestinal diseases (such as IBS). Tibble et al. [10] included 602 patients with symptoms of digestive tract discomfort, and found that patients with organic intestinal disease had significantly higher FC concentration than patients with functional bowel disease. The sensitivity and specificity of using FC to distinguish organic intestinal disease from the functional bowel disease were $83 \%$ and $79 \%$, respectively (cut-off value was 10 $\mathrm{mg} / \mathrm{L}$ ). Subsequently, Rheenen [11] included 754 patients for meta-analysis, and concluded that the combined sensitivity of the use of FC to distinguish between IBD and IBS was $80.0 \%$, and the combined specificity was $76 \%$, of which CD subgroup patients (sensitivity: $83 \%$, specificity: $85 \%$ ) was slightly higher than that of the UC subgroup (sensitivity: $72 \%$, specificity: $74 \%$ ).

In this study, no matter using ELISA or FEIA, the FC concentration of patients with functional bowel disease was significantly lower than that of patients with clinical remission or active IBD. While using FEIA, the optimal cut-off point of FC concentration was $131.79 \mu \mathrm{g} / \mathrm{g}$. At this time, the AUC of the method 
for identifying IBD and functional bowel disease was 0.881 , the sensitivity was $81.82 \%$, and the specificity was $80.77 \%$. The ROC curve analysis results of ELISA are similar to that of FEIA. According to the best cut-off value obtained from the ROC curve, the positive rates of the two methods were not statistically different. The above results suggest that the immunofluorescence assay developed by us is equivalent to the clinical application of FC classic ELISA in the differential diagnosis of IBD and functional bowel disease.

According to the research on FC identification of IBD and IBS, the best cut-off value of FC concentration after ROC curve analysis is set to $8-150$ $\mathrm{mg} / \mathrm{kg}$, the sensitivity is about $83 \%-100 \%$, and the specificity is about $51 \%-$ $100 \%$ [12]. The experimental results of this study are similar to those studies. However, in recent years, with the improvement of the FC detection method, the sensitivity and specificity of the FC discriminator for organic intestinal disease and functional bowel disease have been increasing, almost all of which are close to $95.0 \%$. Von Roon et al. [13] included a total of 5983 patients in 30 studies for meta-analysis. The FC concentration of patients with IBD was significantly higher than that of patients with IBS. The sensitivity and specificity of assisting diagnosis were $95 \%$ and 91\%, respectively. Later, Van Rheenen et al. [11] included 670 adults and 371 children with suspected IBD for study. Finally, 32\% of adults and $61 \%$ of children were diagnosed as IBD by endoscopy and histopathology. The combined sensitivity and specificity by FC detection were $93 \%$ and 96\%, respectively. Although the results of FEIA and ELISA in the differential diagnosis of IBD and functional bowel disease in this study are similar, the sensitivity and specificity are slightly lower than the recent studies. This may be related to our experimental design and small sample size. We need to further expand the sample size and design different experimental protocols to further evaluate the value of this immunofluorescence assay in the differential diagnosis of IBD and functional bowel disease.

The age comparison showed that the mean age of the IBD group (34.74 \pm 15.61 years) was lower than that of the control group ( $45.00 \pm 16.00$ years), and the difference was statistically significant. Further subgroup analysis found that the mean age of the UC group (44.94 \pm 14.09 years) was not different from the control group, mainly because the mean age of the CD group $(25.30 \pm 10.08$ years old), suggesting the average age of patients in the IBD group lower than the control group was mainly caused by the small average age of the included CD patients. However, this is consistent with the epidemiological characteristics of IBD [14], because CD patients usually occur in adolescents and young people. At the same time, the current literature only mentions that the neonatal FC value is higher, and gradually decreases with age, and reaches the normal level and remains stable at the age of 5 [15]. This FC change in infants and young children may be related to the establishment of intestinal flora in childhood. At present, it is not found that adult age affects the concentration of FC. Therefore, this study concluded that the age difference between the IBD group and the control group had no significant effect on the FC values of the two groups. 
In this study, the FC concentration measured by the two detection methods could not effectively distinguish between IBD patients with remission, mild, moderate, or severe active stage. This may be related to the fact that the existing symptom score system of IBD is not completely true and cannot effectively reflect the true intestinal inflammation of IBD. Colonoscopy is the gold standard for detecting intestinal inflammation. The CDAI score, Mayo clinical score, UCAI score and other symptom scoring systems were in poorly consistent with the endoscopic scoring system of IBD, while FC is an indicator that specifically reflects intestinal inflammation. We need to further analyze the relationship between FC and IBD endoscopic inflammation degree and recurrence in subsequent studies.

\section{Conclusion}

In summary, our data indicated that FC concentration determined by FEIA can effectively distinguish IBD from functional bowel disease, providing a non-invasive index for screening intestinal inflammation, with broad application prospects in bowel diseases.

\section{Acknowledgements}

This work is supported by grants from the National Natural Science Foundation of Guangdong (2017A030313893), Guangzhou Science and Technology Plan (201607010074).

\section{Conflicts of Interest}

The authors declare no conflicts of interest regarding the publication of this paper.

\section{References}

[1] Zeng, Z., Zhu, Z., Yang, Y., et al. (2013) Incidence and Clinical Characteristics of Inflammatory Bowel Disease in a Developed Region of Guangdong Province, China: A Prospective Population-Based Study. Journal of Gastroenterology and Hepatology, 28, 1148-1153. https://doi.org/10.1111/jgh.12164

[2] APDW 2004 Chinese IBD Working Group (2006) Retrospective Analysis of 515 Cases of Crohn's Disease Hospitalization in China: Nationwide Study from 1990 to 2003. Journal of Gastroenterology and Hepatology, 21, 1009-1015. https://doi.org/10.1111/j.1440-1746.2006.04140.x

[3] Wang, Y. and Ouyang, Q. (2007) Ulcerative Colitis in China: Retrospective Analysis of 3100 Hospitalized Patients. Journal of Gastroenterology and Hepatology, 22, 1450-1455. https://doi.org/10.1111/j.1440-1746.2007.04873.x

[4] Roseth, A.G., Schmidt, P.N. and Fagerhol, M.K. (1999) Correlation between Faecal Excretion of Indium-111-Labelled Granulocytes and Calprotectin, a Granulocyte Marker Protein, in Patients with Inflammatory Bowel Disease. Scandinavian Journal of Gastroenterology, 34, 50-54. https://doi.org/10.1080/00365529950172835

[5] Kalantari, H., Taheri, A., Yaran, M., et al. (2015) Fecal Calprotectin Is a Useful Marker to Diagnose Ulcerative Colitis from Irritable Bowel Syndrome. Advanced 
Biomedical Research, 4, 85. https://doi.org/10.4103/2277-9175.156647

[6] Alibrahim, B., Aljasser, M.I., Salh, B., et al. (2015) Fecal Calprotectin Use in inflammatory Bowel Disease and Beyond: A Mini-Review. Canadian Journal of Gastroenterology and Hepatology, 29, 157-163. https://doi.org/10.1155/2015/950286

[7] The Society of Gastroenterology of the Chinese Medical Association (2012) Consensus on the Diagnosis and Treatment of Inflammatory Bowel Disease (2012-Guangzhou). Chinese Journal of Gastroenterology, 17, 763-781.

[8] Peyrin-Biroulet, L., Loftus, E.V. Jr, Harmsen, W., et al. (2010) Cumulative Incidence of and Risk Factors for Major Abdominal Surgery in a Population-Based Cohort of Crohn's Disease. Gastroenterology, 128, S1184.

[9] Song, X.M., Gao, X., Li, M.Z., Chen, Z.H., Chen, S.C., Hu, P.J., et al. (2011) Clinical Features and Risk Factors for Primary Surgery in 205 Patients with Crohn's Disease: Analysis of a South China Cohort. Diseases of the Colon \& Rectum, 54, 1147-1154. https://doi.org/10.1097/DCR.0b013e318222ddc3

[10] Tibble, J.A., Sigthorsson, G., Foster, R., Forgacs, I. and Bjarnason, I. (2002) Use of Surrogate Markers of Inflammation and Rome Criteria to Distinguish Organic from Nonorganic Intestinal Disease. Gastroenterology, 123, 450-460.

https://doi.org/10.1053/gast.2002.34755

[11] Van Rheenen, P.F., Van de Vijver, E. and Fidler, V. (2010) Faecal Calprotectin for Screening of Patients with Suspected Inflammatory Bowel Disease: Diagnostic Meta-Analysis. BMJ, 341, c3369. https://doi.org/10.1136/bmj.c3369

[12] Papadia, C., Maffei, E., Del Rio, P., Taylor, S., Caini, S., Montana, C., et al. (2013) Sensitivity and Specificity of Magnetic Resonance Enterography in the Clinical Management of Fistulizing Crohn's Disease. Inflammatory Bowel Diseases, 19, 1896-1903.

[13] Von Roon, A.C., Karamountzos, L., Purkayastha, S., et al. (2007) Diagnostic Precision of Fecal Calprotectin for Inflammatory Bowel Disease and Colorectal Malignancy. American Journal of Gastroenterology, 102, 803-813. https://doi.org/10.1111/j.1572-0241.2007.01126.x

[14] Ng, S.C., Tang, W., Ching, J.Y., Wong, M., Chow, C.M., et al. (2013) Incidence and Phenotype of Inflammatory Bowel Disease Based on Results from the Asia-Pacific Crohn's and Colitis Epidemiology Study. Gastroenterology, 145, 158-165. https://doi.org/10.1053/j.gastro.2013.04.007

[15] Rugtveit, J. and Fagerhol, M.K. (2002) Age-Dependent Variations in Fecal Calprotectin Concentrations in Children. Journal of Pediatric Gastroenterology and Nutrition, 34, 323-325. https://doi.org/10.1097/00005176-200203000-00022 